\title{
Tensile force effect of pelvic belt in patients with antenatal symphyseal pain
}

\author{
Fayiz El-Shamy ${ }^{1,}$, Magda Morsy², Anees Saleh $^{3}$ \\ ${ }^{1}$ Department of Physical Therapy for Women's Health, Faculty of Physical Therapy, Kafelsheikh University, Kafelsheikh, Egypt \\ ${ }^{2}$ Department of Physical Therapy for Gynecology and Obstetrics, Faculty of Physical Therapy, Cairo University, Cairo, Egypt \\ ${ }^{3}$ Department of Biomechanics, Faculty of Physical Therapy, Kafelsheikh University, Kafelsheikh ,Egypt
}

\section{Email address:}

ff_elshamy@yahoo.com (F. El-Shamy),ANEESPT@yahoo.com (A. Saleh)

\section{To cite this article:}

Fayiz El-Shamy, Magda Morsy, Anees Saleh. Tensile Force Effect of Pelvic Belt in Patients with Antenatal Symphyseal Pain. European Journal of Preventive Medicine. Vol. 2, No. 6, 2014, pp. 90-94. doi: 10.11648/j.ejpm.20140206.12

\begin{abstract}
Aims: To investigate the effect of pelvic belt on relieving antenatal symphyseal pain. Study design: A randomized clinical controlled trial. Place and Duration of study: Department of Obstetrics, Kafelsheikh general hospital - Kafelsheikh, between June 2014 and September 2014. Methodology: Twenty pregnant women (age range 25-30 years) at 24 week $^{s}$ gestation with a good general health were selected randomly, clinically diagnosed with symphyeal pain and divided into 2 equal groups (A\&B). Patients in group A were treated by pelvic belt with tensile force of $50 \mathrm{~N}$. for 4 weeks, patients in group B without any treatment. Severity of symphyseal pain and diastasis were measured by mean of a $100-\mathrm{mm}$ horizontal visual analogue scale and ultrasound before and after the treatment program. Results: Patients in group A showed non significant difference in severity of symphyseal pain and diastasis $(\mathrm{P}=0.54 \& 0.84$ respectively). Patients in group $\mathrm{B}$ showed highly significant increase in severity of symphyseal pain and diastasis $(\mathrm{P}=0.001)$. There were highly significant difference between both groups $(\mathrm{P}=0.001)$ after the treatment program. The symphyseal pain value was significantly correlated with the decrease in symphyseal diastasis.(Pearson's correlation coefficient $0.59, \mathrm{P}=0.005$ ). Conclusion: Application of a pelvic belt at the symphysis pubis decrease antenatal symphyseal diastasis and pain.
\end{abstract}

Keywords: Pregnancy, Symphyseal Pain, Tensile Force, Pelvic Belt

\section{Introduction}

The pubic symphysis is a nonsynovial amphiarthrodial joint, and comes from the Greek word "symphysis", meaning "growing together". The width of the pubic symphysis at the front is $3-5 \mathrm{~mm}$ greater than its width at the back [1]. From biomechanical point of view, analysis of the pelvis shows the skinny regions function as arches, transferring the weight of the upright trunk from the sacrum to the hips. The symphysis pubis connects these two weight-bearing arches, and the ligaments that surround this pelvic region maintain the mechanical integrity [2]. Biomechanically ,the main motions of the symphysis pubis are superior/inferior glide and separation/compression. The functions of the joint are to absorb shock during walking and allow delivery of a baby [3]. During pregnancy in the human there are some biomechanical changes which occur inside symphysis pubis, for example hormones such as relaxin remodel this ligamentous capsule allowing the pelvic bones to be more flexible for delivery. The gap of the symphysis pubis, normally is $4-5 \mathrm{~mm}$ but during pregnancy there will be an increase of at least 2-3 $\mathrm{mm}$, therefore, it is considered that a total width of up to $9 \mathrm{~mm}$ between the two bones is normal for a pregnant woman [4]. The symphysis pubis separates to some degree during childbirth. In some women this separation can become a diastasis of the symphysis pubis. The diastasis could be the result of a rapid birth [5]. or a forceps delivery. [6] or maybe even be prenatal [7]. A diastasis of the symphysis pubis is a symptom of pelvic girdle pain (PGP). Overall, about $45 \%$ of all pregnant women and $25 \%$ of all women postpartum suffer from PGP [8].Symphysis pubis dysfunction is recognized as being a common problem and an incidence of $1: 36$ to $1: 37$ during pregnancy have been reported [9]. It is a significant problem for some women because they often face a major functional difficulties resulting in a considerable decrease in quality of life. Symphyeal pain occurs due to symphysis pubis 
diastasis (SPD) means an abnormally gap between the two pubic bones at the symphysis pubis joint situated in the front of the pelvis which increases pain and tenderness in the pubic area [10].This gap due to the fact that ligaments which 'tie' the joint become slightly slacker under the influence of the pregnancy hormones [11]. The separation of more than $1 \mathrm{~cm}$ is pathologic with rupture of the symphyseal ligaments, symphysis pubis become unstable. If the joint is not firmly 'tied' by all its ligaments, it cannot effectively perform its role and excessive strain is placed on all the pelvic joints giving rise to all painful symptoms and difficulty in movement [12]. Sever pelvic pain during pregnancy and after delivery was strongly associated with increasing symphyseal diastasis ,such pain may not only be limited to the current pregnancy, since early onset and high intensity of pain are reported as risk factors of repeated and worsening pain after labor and in subsequent pregnancies and in post-partum period [13]. Objective criteria with respect to cause as well as pain evoking structures are difficult to find which makes optimal choice of therapy uncertain or even controversial. Many patients experience relieve of pain when using a pelvic belt, which makes the use to a common part of the therapy $[14,15,16]$. The underlying theory of the use of a pelvic belt is that the articular surfaces of the symphysis pubis will be pressed together and/or that the symphysis pubis will be placed in an extreme position to provide stability, but there is no in vivo proof of this mechanical effect $[17,18,19]$. The need of this study developed from the lack in the quantitative knowledge and information in the published studies about the ideal modality in treating sympyseal pain [20]. So, this study was designed to assess the effect of pelvic belt on relieving antenatal symphyseal pain.

\section{Materials and Methods}

Study design and sampling following the hospitals ethical committee approved the study at obstetric department of Kafelsheikh general hospital and written informed consent was obtained from each subject. 20 pregnant women at 24 week's gestation with a good general health and symphyseal pain were recruited according to the inclusion and exclusion criteria of the study. The main exclusion criterion was the presence of low back and/ or pelvic pain before pregnancy. Also excluded were subjects with pain radiating to below the knee, known congenital anomalies of the spine, known rheumatic disease affecting the locomotion system or previous surgery of the lumbar spine or pelvis. The participants were randomly assigned to one of the two groups; study group (A) and a control group (B). through a block-style randomization scheme [21]. Their age ranged from (25-30) years old, number of parity ranged from ( 2-3 ) and body mass index (BMI) would not exceed $30 \mathrm{~kg} / \mathrm{m}^{2}$. The design of this study was a clinical controlled trial. The study was done from June 2014 to September 2014. Before intervention, severity of pain was measured by mean of a $100-\mathrm{mm}$ horizontal visual analogue scale for the worst pain during the preceding week (VAS) with a range from 0 to $100 \mathrm{~mm}$, where 0 denoted no pain and 100 worst possible pain [22]. Ultrasound had been used to estimate amount of separation between two symphysis pubic bones (Medison apparatus x 6). The same re evaluation was performed after 4-weeks for both groups (A\&B). Group A: Consisted of ten women, treated by pelvic belt for 4 weeks, each woman would be asked to wear the pelvic belt all the day except during sleep or having a shower. A belt of non-elastic material was used (model 3221/3300, Rafys, Hengelo, The Netherlands) $5 \mathrm{~cm}$ wide at the anterior and $7 \mathrm{~cm}$ at the posterior side. The belt was adjusted at the level of the symphysis. The applied tensile force of the belt was $50 \mathrm{~N}$, because $50 \mathrm{~N}$ was the minimum tension needed to influence SIJ laxity[23,24]. Group B: Consisted of ten women without any treatment.

\subsection{Data Analysis}

Statistical analysis involved the calculation of the means and standard deviations for each of the variables measured. Paired t-tests were used to evaluate within-group changes. Differences between the two groups were assessed using an independent samples t-test. The correlation between the symphyseal pain and distension was determined by calculating the Pearson's correlation coefficient. A P-value of $<0.05$ was taken to represent statistical significance. Data analysis was performed using SPSS software version 16 .

\section{Results}

All data had been collected and statistically analyzed and presented under the following headings;

\subsection{General Characteristics of the Patients}

In this study, 20 participants were divided randomly into two equal groups (A\&B). Group (A): 10 participants were included in this group; the mean age and BMI were $(27.33 \pm 1.09)$ years and $(28.3 \pm 1.09) \mathrm{Kg} / \mathrm{m}^{2}$. Group (B): 10 participants were included in this group; the mean age and BMI were $(26.16 \pm 1.01)$ years and $(27.9 \pm 1.45) \mathrm{Kg} / \mathrm{m}^{2}$. There was no significant difference $(\mathrm{P}=0.73$ and 0.85$)$ between both groups $(\mathrm{A} \& \mathrm{~B})$ regarding their ages and BMI respectively.

\subsection{Symphyseal Pain Intensity on Visual Analogue Scale (mm)}

Group A: There was no significant difference in participant's symphyseal pain intensity $(\mathrm{P}=0.54)$, as the mean values of pain intensity was $53.8 \pm 13.03$ at the beginning of the study, while it was $48.28 \pm 12.3$ at the end of the study.

Group B: There was significant increase in participant's symphyseal pain intensity $(\mathrm{P}=0.001)$, as the mean values of pain intensity was $54.1 \pm 14.2$ at the beginning of the study, while it was $63.5 \pm 9.8$ at the end of the study.

There was no significant difference in participant's symphyseal pain intensity $(\mathrm{P}=0.62)$ at the beginning of the study. But there was significant increase $(\mathrm{P}=0.001)$ at the end of the study between both groups( A\&B).(Table 1\& Fig.1). 
Table (1). Mean values of Symphyseal Pain intensity for participants in both groups $(A \& B)$.

\begin{tabular}{lllll}
\hline & \multicolumn{4}{l}{ Symphyseal Pain intensity $(\mathbf{m m})$} \\
\cline { 2 - 5 } & Group A & \multicolumn{3}{l}{ Group B } \\
\cline { 2 - 5 } & Pre & Post & Pre & Post \\
\hline Mean & 53.8 & 48.28 & 54.1 & 63.5 \\
\pm SD & \pm 13.03 & \pm 12.3 & \pm 14.2 & \pm 9.8 \\
P. value & 0.5 & 0.001 & & \\
Significance & & $\mathrm{S}$ & & \\
\hline
\end{tabular}

SD: Standard Deviation, P. value: probability value, NS: Non Significant, S: Significant.

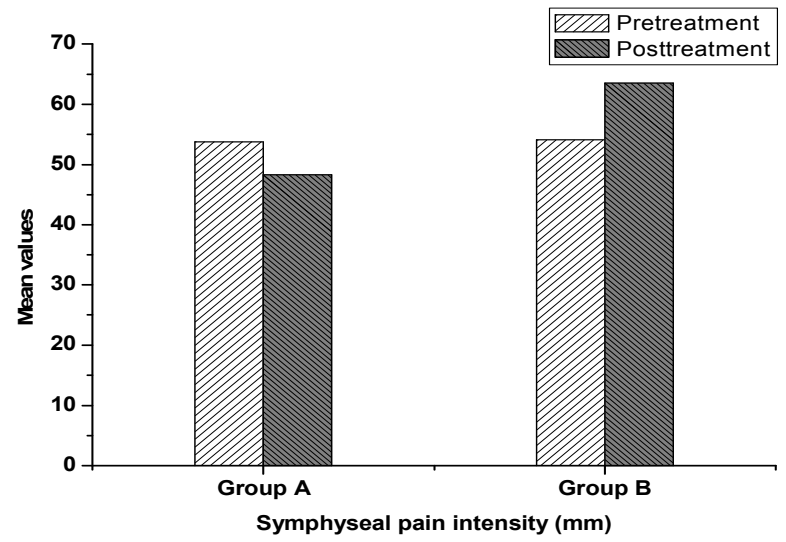

Figure 1. Mean values of Symphyseal Pain intensity for participants in both $\operatorname{groups}(A \& B)$

\subsection{Symphyseal Diastasis (cm)}

Group A: There was no significant difference in participant's symphyseal diastasis $(\mathrm{P}=0.84)$, as the mean values of the symphyseal diastasis was $1.91 \pm 0.21$ at the beginning of the study, while it was $1.93 \pm 0.3$ at the end of the study.

Group B: There was significant increase in participant's symphyseal diastasis $(\mathrm{P}=0.001)$, as the mean values of the symphyseal diastasis was $1.97 \pm 0.41$ at the beginning of the study, while it was $2.82 \pm 0.2$ at the end of the study.

There was no significant difference in participant's symphyseal diastasis $(\mathrm{P}=0.73)$ at the beginning of the study. But there was significant increase $(\mathrm{P}=0.001)$ at the end of the study between both groups (A\&B) (Table 2\& Fig. 2).

Table (2). Mean values of Symphyseal diastasis for participants in both groups $(A \& B)$.

\begin{tabular}{|c|c|c|c|c|}
\hline & \multicolumn{4}{|c|}{ Symphyseal diastasis(cm) } \\
\hline & \multicolumn{2}{|c|}{ Group A } & \multicolumn{2}{|c|}{ Group B } \\
\hline & Pre & Post & Pre & Post \\
\hline Mean & 1.91 & 1.93 & 1.97 & 2.82 \\
\hline$\pm \mathrm{SD}$ & \pm 0.21 & \pm 0.3 & \pm 0.41 & \pm 0.2 \\
\hline p. value & 0.84 & 0.001 & & \\
\hline Significance & NS & S & & \\
\hline
\end{tabular}

SD: Standard Deviation, P. value: probability value, NS: Non Significant, S: Significant.

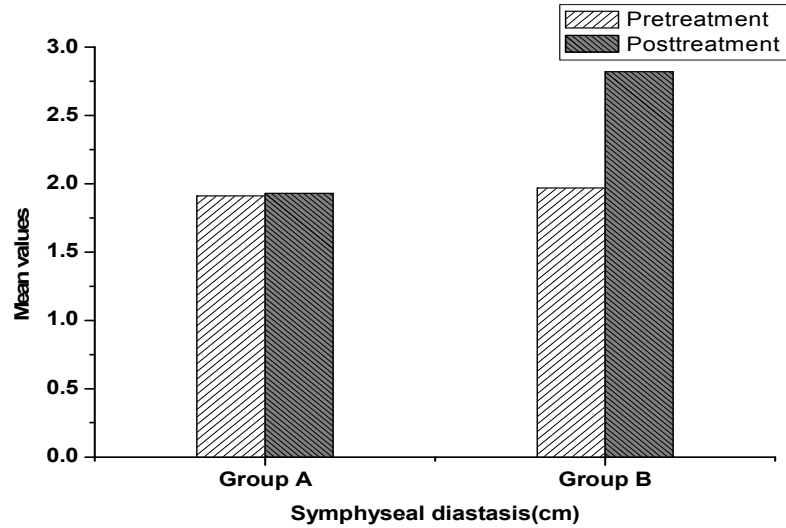

Figure 2. Mean values of Symphyseal diastasis for participants in both groups $(A \& B)$

Correlation between Symphyseal diastasis and Pain:

The decrease in mean symphyseal pain value with the application of pelvic belt at the symphysis pubis compared with the condition without belt was significantly correlated with the decrease in symphyseal diastasis.(Pearson's correlation coefficient $0.59, \mathrm{P}=0.005$ ).

\section{Discussion}

In this study the effect of pelvic belt on symphyseal diastasis and pain was studied in 20- patients with antenatal symphyseal pain, symphyseal diastasis was restricted by application of pelvic belt (with tensile force of $50 \mathrm{~N}$ ) at the level of symphysis pubis which significantly decrease the symphyseal laxity and pain intensity at study group compared with control group. The results of the study come in agreement with Ho et al [25] this review critically evaluates the effectiveness of maternity support belts in the treatment of pelvic pain. Wearing maternity support belts may be beneficial for pain relief and improved functional status in patients with pelvic pain. The use of maternity support belts may improve lumbo-pelvic stability by press the articular surfaces of symphysis pubis and sacroiliac joint and place them in their extreme position to provide stability. The strain of the ligaments in pelvis is often assumed to cause pelvic pain which results from a combination of factors including hormonal effects, altered posture, muscle weakness. Also, ligamentous laxity may render mechanical instability which further increases stretch and strain on the pelvic and thus stimulates mechanoreceptors which anocicptive function leading to pain. So, an increase in joint stability would therefore leads to pain reduction and reverse all previous actions[26]. A pelvic belt may be used in the treatment of instability of both sacroiliac joints and symphysis pubis. The use of pelvic belt reduces the ability of the in nominates bones of woman to be pushed out worldly from each other during movement. Application of a pelvic belt significantly decreases mobility of the symphysis pubis and sacroiliac joints[11]. Mens et al [27] reported that a pelvic belt was effective in management of symphyseal pain but was less effective during pregnancy than after delivery. The pelvic belt was believed to 
have a stabilization effect as it might stimulate the actions of different local stabilizers. For instance, a pelvic belt worn in a high position may stimulate the action of the transversus abdominis by the anterior compression on the anterior superior iliac spine and stimulate the action of multifidus by the posterior compression on the posterior superior iliac spine. In a low position, it may stimulate the action of pelvic floor muscles. Also, recent studies showed that lumbo- pelvic stability was achieved through specific training of the transverses abdominals, multisided and pelvic floor muscles.

\subsection{Limitations}

Although the employed design of the present study (a randomized controlled clinical trial) is of importance the recruited small sample size could be a potential limitation of the current study. Based on sample size estimation with the power of the study $1-\mathrm{B}=80 \%$, and in order to detect the effect size of $d=0.5$ with a significance level of a $<0.05,50$ participants were needed for each group. At present it is not possible to quantify symphysial diastasis values in loaded position. By measuring in supine position, we measured the influence of the pelvic belt on symphysial diastasis and tried to exclude muscle activity and tension of ligaments that could have contributed to the decreasing symphysial diastasis. Further studies will focus on the performance of measurements in standing position with the aim to investigate how symphysial laxity will behave in the standing position with and without a pelvic belt. Lack of an objective outcome measure such as measuring pain intensity by VAS is another limitation of the present study.

\section{Conclusion}

Application of a pelvic belt at the symphysis pubis significantly decrease antenatal Symphyseal diastasis and Pain. The results might be the basis for clinical studies about the use of pelvic belts in pregnancy-related pelvic girdle pain.

\section{Acknowledgements}

The authors thank the physicians, nurses and patients of obstetric department at Kafelsheikh general hospital for participating in this study.

\section{References}

[1] Becker, I., Woodley, S. J. and Stringer, M. "The adult human pubic symphysis: a systematic review". Journal of Anatomy. 217(5):475-87(2010).

[2] Rodrigo, C. and Renato, P."Nutrition pathways to the symphysis pubis". Journal of Anatomy. 204(3), 209-215, 2004.

[3] Gamble, J. and Simmons, S. "The Symphysis Pubis: Anatomic and Pathologic Considerations". Clinical Orthopaedics and Related Research.203, 261-272,1986.

[4] Kelly, O., Anne, P. and Gerald, M."Pubic symphysis separation". Fetal and Maternal Medicine Review 13, 141-155, 2002.

[5] Panditrao, S. , Eknathrao, B. , Popat, G. and Ramkrishna, M. ,Pubic Symphysial Diastasis During Normal Vaginal Delivery", Journal of Obste India. 55 (4),365-366, 2005.

[6] Scicluna, J., Alderson, J., Webster, V. and Whiting, P. "Epidural analgesia for acute symphysis pubis dysfunction in the second trimester". International Journal of Obstetric Anesthesiology. 13(1),50-2, 2004.

[7] Wu, W. H., Meijer, O. G., Uegaki, K., Mens, J. M. A., van Dieën, J. H., Wuisman, P. I. J. M. and Östgaard. H. C. "Pregnancy-related pelvic girdle pain (PPP), I: Terminology, clinical presentation, and prevalence". European Spine Journal. 13( 7 ), 2004.

[8] Vleeming ,A., Albert ,H. and Ostgaared, H., "European guidelines for the diagnosis and treatment of pelvic girdle pain". Eur. Spine. J.17,794-819, 2008.

[9] Wellock, K. and Cricbton, A., "Understanding pregnant women experience of symphysis pubis dysfunction: the effect of pain". Evidence based midwifery. 5(2),40-46, 2007.

[10] Leadbetter, R., Mawerd, D. and Lindow, S., "Symphysis pubis dysfunction: a review of the literature". The Journal of Maternal-Fetal and Neonatal Medicine.16,349-354,2004.

[11] Okkafor, U. and Sokunbi, T., Physiotherapy Management of Sub-acute Postpartum Diastasis of Pubic Symphysis A case report". Journal of the Nigeria society of physiotherapy.17,37-40, 2009.

[12] Khorashadi, L., Petscavage, J. and Richardson, M., "Postpartum symphysis pubis diastasis" . Radiological Case Reports. 6(3), 2011.

[13] Bjourklund, K., Bergstrom, S. and Nordostrom, M., "Symphyseal distension in relation to serum relaxin levels and pelvic pain in pregnancy".Acta.Obstet.Gynecol.Scand.79(4),269-275, 2000.

[14] Dunbar, R., Puerperal diastasis of the public symphysis". A case report.J.Reprod.Med.47,581-3, 2002.

[15] Berg, G., Hammar, M., Mo"ller-Nielsen, J., Linde'n, U. and Thorblad, J., "Low back pain during pregnancy". Obstet. Gynecol. 71,71-75,1988.

[16] Mens, J.M.A., Vleeming, A., Stoeckart, R., Stam, H.J. and Snijders, C.J., "Understanding peripartum pelvic pain. Implications of a patient survey". Spine 21,1363-1370, 1996.

[17] O"stgaard, H.C., Zetherstro"m, G., Roos-Hansen, E. and Svanberg, B., Reduction of back and posterior pelvic pain in pregnancy". Spine 19, 894-900,1994.

[18] Richordson, C., Snijders, C., Hides, J. and Damen, 1. "The relation between the transverse abdominis muscles, sacroiliac joint mechanics, and low back pain", Spine.27,399-405,2002.

[19] Snijders, C.J., Vleeming, A. and Stoeckart, R., "Transfer of lumbosacral load to iliac bones and legs. Part I: biomechanics of self-bracing of the sacroiliac joints and significance for treatment and exercise". Clin. Biomech. 8, 285-294,1993a.

[20] Snijders, C.J., Vleeming, A. and Stoeckart, R., "Transfer of lumbosacral load to iliac bones and legs. Part 2: loading of the sacroiliac joints when lifting in a stooped posture". Clin. Biomech. 8, 295-301,1993b. 
[21] Piantadosi,s. "Clinical trial: a methodologic perspective. John wily\& sons, new York. 1997.

[22] Collins, S.L., Moore, R.A. and McQuay, H.J. "The visual analogue pain intensity scale: what is moderate pain in millimeters?"' Pain. 72, 95-97,1997.

[23] Damen, L., Spoor, C.W., Snijders, C.J.and Stam, H.J. “Does a pelvic belt influence sacroiliac joint stiffness?: Clin. Biomech. 17,495-498, 2002.

[24] Mens, J.M., Vleeming, A., Snijders, C.J., Stam, H.J.and Ginai, A.Z. "The active straight leg raising test and mobility of the pelvic joints". Eur. Spine J. 8, 468-473,1999.
[25] Ho, S., Yu, w., Lae, T. and Chow, D. "Effectiveness of maternity support belts in reducing low back pain during pregnancy :A Review ". Journal of clinical nursing.18(11),1523-1532,2008.

[26] Lee, y. and Chein, C. "Lumbar vertebral angles and back muscle loading with belts", Industrial Health,37,390-397,1999.

[27] Mens, J., Damen, L., Snijders ,C. and Stam, S. "The mechanical effect of a pelvic belt in patients with pregnancy related pelvic pain", Clinical biomechanics. 21.122-127,2006. 